\title{
Increasing Emotion regulation skills with dialectical behavior therapy skills training: A single-case study on an elementary school student with borderline intellectual functioning
}

\author{
Kara Andrea Handali ${ }^{\mathrm{a}}$ and Linda Primana ${ }^{\mathrm{b}^{*}}$ \\ ${ }^{a}$ Faculty of Psychology, Universitas Indonesia, Depok, Indonesia $;{ }^{b}$ Educational Psychology \\ Department, Faculty of Psychology, Universitas of Indonesia, Depok, Indonesia \\ *Corresponding Author: \\ Linda Primana \\ Educational Psychology Department \\ Faculty of Psychology, Universitas Indonesia \\ Jl. Lkr. Kampus Raya, Depok, Jawa Barat \\ Indonesia, 16424 \\ Tel.: +62 217270004 \\ e-mail address: primana.linda@gmail.com
}




\title{
Increasing emotion regulation skills with Dialectical Behavior Therapy skills training: A single-case study on an elementary school student with borderline intellectual functioning
}

\begin{abstract}
Individuals with borderline intellectual functioning (BIF) are persons with an IQ between 70 and 85 points and characterized by their wide range of cognitive and social difficulties. During their school-age years, most of them have problems with school adjustment. Their ability to regulate emotion is limited due to poor executive function. This condition affects both their behavior in the classroom and their social relations. Dialectical behavior therapy (DBT), developed by Linehan in 1993, can effectively increase emotion regulation skills in adolescents and adults with intellectual disability, including BIF. This work was a single-case study to examine the effectivity of DBT skills training, one of four modes in DBT, in increasing emotion regulation skills in elementary school children with BIF. An A-B-A design was used in this study, with all three phases completed in 24 days. The program consisted of two adapted DBT modules: mindfulness and distress tolerance. Parents and teachers were also involved to enhance the treatment effects. Data were analyzed through visual and statistical analyses. Results of the study showed a significant difference in emotion regulation skills before and after the intervention $(\mathrm{Z}=-2.023, p<0.05, \mathrm{r}=-0.64)$. Through DBT skills training, the subject gained knowledge and skills about emotion regulation. These outcomes were achieved by adapting the module to suit the characteristics of BIF. The findings suggest that adapted DBT is a promising intervention to improve emotion regulation skills in children with BIF.
\end{abstract}

Keywords: borderline intellectual functioning; dialectical behavior therapy; elementary school students; emotion regulation skills

\section{Introduction}

An individual is classified as having borderline intellectual functioning (BIF) if he or she has an IQ score between 70 and 85 (American Psychological Association, as cited in Baglio et al., 2016). Many children with BIF have problem with school adjustment, both academically and socially. Their cognitive deficit is characterized by their limited executive function (Van Nieuwenhuijzen \& Vriens, 2012), which leads to a wide range of difficulties in the classroom, including understanding abstract concepts; generalizing skills, knowledge, and strategies; organizing new material; and assimilating new information (Shaw, 2010). These characteristics cause children with BIF to struggle in acquiring basic academic skills, such as reading, writing, and arithmetic; this difficulty often results in low academic achievement (Karande, Kanchan, \& Kulkarni, 2008). Besides having academic problems, the poor executive function of children with BIF limits their capacity to understand social cues and show appropriate responses under some social conditions. They often show aggressive and anti-social behavior (Van Nieuwenhuijzen \& Vriens, 2012) that causes them to be rejected by peers (Jankowska, 2016). McClure, Halpern, Wolper, and Donahue (2009) explained that these behaviors are due to their lack of emotion regulation skills, also known as emotion dysregulation.

Emotion regulation is the ability to modulate emotional arousal in an adaptive way (Graziano, 
Reavis, Keane, \& Calkins, 2007), such as adjusting actions, verbal responses, or nonverbal expressions to fit normative conditions (Linehan, 2015). It allows us to handle stressful situations, such as learning new materials and building positive social relationships (Boulanger, 2015). With their poor executive function, children with BIF tend to have difficulties in interpreting social situations (Masi, Marcheschi, \& Pfanner, 1998 as cited in Van Nieuwenhuijzen \& Vriens, 2012). Thus, they often misinterpret emotions and show inappropriate emotions (Dekker, Koot, Van der Ende, \& Verhulst, 2002; Einfeld \& Tonge, 1996 as cited in Van Nieuwenhuijzen \& Vriens, 2012; Boulanger, 2015). In coping with emotions, children with BIF use emotion regulation strategies that provide immediate emotional release but make a negative long-term impact, such as aggressive behaviors (Van Nieuwenhuijzen \& Vriens, 2012).

Emotion dysregulation in children with BIF is not only related to their problem in socializing with peers but also to their behavior in the classroom. Davis and Levine (2013) reported that cognitive emotion regulation skills, also known as cognitive reappraisal, can effectively help children change their thoughts of negative emotions into a neutral one, so that they can attend to the study materials. Unfortunately, cognitive deficits in children with BIF make it difficult for them to conduct cognitive reappraisal (Boulanger, 2015), which limits their learning process. Therefore, increasing emotion regulation skills in children with BIF is important to improve their learning process.

Cognitive behavioral therapy (CBT) has been shown to effectively improve emotion regulation skills in clients with intellectual disability, including BIF (McClure et al., 2009; McWilliams, 2014). However, CBT is effective for this population only if it includes relaxation, selfmonitoring, video modeling, and other strategies that are classified as mindfulness-based strategies (McWilliams, 2014; Sturmey, 2004; Whitaker, 2001). Rather than emphasizing cognitive changes, clients learn to regulate their emotion through mindfulness, or "the ability to consciously experience and observe one-self and surrounding events with curiosity and without judgement ... to participate in the flow of the present moment effectively" (Linehan, 2015). Many studies have shown that dialectical behavior therapy (DBT), a mindfulness-based therapy, is effective to improve emotion regulation skills in adolescents and adults with intellectual disability (Brown, Brown, \& Dibiaso, 2013; Charlton \& Dykstra, 2011; Florez \& Bethay, 2017; Lew, Matta, Tripp-Tebo, \& Watts, 2006; McWilliams, 2014). By contrast, the effectivity of DBT has yet to be proven in school-aged children with intellectual disability.

DBT is a modified form of CBT that is based on mindfulness strategies; it was developed by Linehan in 1993 to treat emotion dysregulation (Linehan, 2015). Unlike a standard CBT, DBT is not only focused in cognitive and behavioral changes but also emphasizes the importance of accepting the emotional experience (Linehan, 2015). The dialectical approach in DBT believes that radical acceptance to emotional experience will lead the client to change his or her behavior (Linehan, 1993 as cited in McMain, Korman, \& Dimeff, 2001). DBT has four modes: individual therapy, phone consultation, team consultation, and skills training (Nickelson, 2013). Skills training is the basic mode of DBT, because it consists of emotion regulation skills that can help the client regulate his or her emotion. This study uses skills training as a sole intervention, as it had been proven effective in the previous research (Linehan, 2015).

DBT skills training involves four modules: mindfulness, distress tolerance, emotion regulation, and interpersonal effectiveness. Mindfulness skill, as the basic skill in DBT, must be given first 
before the client starts learning the other modules. The three other modules should be given individually based on the client's needs (Linehan, 2015). This study only used two modules, namely, mindfulness and distress tolerance, due to the participant's priority need, that is his inability to tolerate stressful situations. The intangible concept of dialectical perspective is challenging for clients with intellectual disability to understand; therefore, using DBT to this population, including BIF, requires some modifications. The modifications are (1) simplified language and concept and increased visual aids, (2) increased feedback, exercise, and repetition, (3) short duration in each session, and (4) environmental support (Brown et al., 2013; Charlton \& Dykstra, 2011; Dykstra \& Charlton, 2003; McNair, Woodrow, \& Hare, 2016).

Previous research demonstrated the effectivity of DBT skills training in adults and adolescents with intellectual disability (Brown et al., 2013; Charlton \& Dykstra, 2011; Florez \& Bethay, 2017), but DBT skills training has not yet been proven effective in school-age children. Given the importance of emotion regulation skills in children with BIF, DBT skills training in this population should be validated. Therefore, this study aimed to examine the effectivity of DBT skills training in increasing emotion regulation skills of an elementary school student with BIF. Unlike standard DBT skills training, which is given to a group of people, this study conducted the training individually due to the client's inability to work in a group. Parent and teachers were also included in the intervention to support the learning process.

\section{Methods}

\section{Participant}

The participant in the study was K, a nine-year-old boy who was a fourth grade student and came from a family of middle-to-high socioeconomic status. His IQ score was 70 (based on Wechsler scale), which categorized him as an individual with BIF. K has problems with his emotion dysregulation that appear when he experienced stressful situations, for example, not being allowed to have recess due to an unfinished task, being pushed to complete the task immediately, or being asked to submit a task he has not done.

\section{Design}

The study used an A-B-A design, which consists of three phases: baseline phase (A), intervention phase (B), and evaluation phase (A'). Baseline phase (A) was the control condition, which was compared with the intervention (B) and evaluation (A') phases. Data measurement was measured for 24 days: 5 days for the baseline phase, 14 days for the intervention phase, and another 5 days for the evaluation phase. The baseline phase consisted of two conditions: three (mid-semester) exam days and two "study-as-usual" days. The intervention phase was conducted every school day during the intervention program. The evaluation phase was given after the DBT skills training finished. All observations were done during the school hours. Randomization was not used in the study because the intervention was constructed based on the DBT skills training structure. Blinding was not used on the participant because emotion regulation development requires a client's awareness, so the participant should know the aim of the intervention. Blinding was also not applicable for parents or teachers because they were involved in the intervention. 


\section{Context}

Observations of target behavior were made during school hours. Another measurement was performed in a different time and location and will be explained in the next section.

\section{Approvals}

This study received qualified approval from the Research Ethics Committee at the Faculty of Psychology of University of Indonesia. Prior to the intervention, informed consent was read, discussed, and signed by the parents (mother), teachers, and the school principal. A learning contract was read, discussed, and signed by the participant.

\section{Measures and Materials}

In this study, the emotion dysregulation response was categorized in two types: verbal and physical. Verbal emotion dysregulation includes making disturbing voices, complaining with a loud voice, arguing with both appropriate/neutral and inappropriate/aggressive words, and screaming. Physical emotion dysregulation includes kicking table/floor, beating own head with hands or other objects, gripping objects (but not throwing it or using it to attack people), showing attacking gesture (with no touching), throwing objects, and attacking people. The target behavior was observed and measured by the researcher, as the therapist in the program, (during baseline and evaluation phase) and teachers (during all phases). The therapist and teachers synchronized the criteria of the target behavior to ensure the reliability of the data given by the teachers in the intervention phase. The appearance of the emotion dysregulation response was presented in percentage due to the characteristic of the target behavior, i.e., only appearing in stressful situations.

The participant's emotion regulation skills were also measured by the emotion regulation checklist (ERC) that was given to significant adults: his mother and two teachers. ERC was developed by Shields and Cicchetti (1997) and has two dimensions, namely, adaptive emotion regulation and lability/negativity. The questionnaire passed several processes: double-translated, approved by two lecturers from the Faculty of Psychology of University of Indonesia, proofread by 33 participants (significant adults), and demonstrated good reliability $(\mathrm{N}=33, \alpha=0.867)$. Data from ERC were processed with SPSS 17. Several materials were used in the intervention: program manual, agenda book, diary cards, emotion regulation skills cards, and stickers for rewards. An audio recorder was also used during the sessions to document the intervention.

\section{Intervention}

The aim of the intervention program was to increase the participant's emotion regulation skills with DBT skills training. Two modules were used in this study, namely, mindfulness and distress tolerance. Mindfulness was given as a basic emotion regulation skill, whereas distress tolerance was selected due to the participant's difficulty in handling stressful situations. Emotion regulation skills are indicated by the participant's ability to use adaptive emotion regulation strategies when he experiences stressful situations. Specifically, mindfulness is operationalized as the "ability to attend to the situations one is in," and distress tolerance is operationalized as the "ability to restrain from showing emotion dysregulation when experiencing stressful situations." Mindfulness skills consist of "States of Mind," "Chain Behavior," "Breathing Exercises," "Check-Your-Tummy Exercises" (modification of observing one's body), and "One Thing at One Time" (focusing on one task before doing other task). Distress tolerance skills consist of the introduction to distress tolerance and several skills to survive in stressful conditions: STOP, 
Exercise on The Spot (modification of scanning body sensations), and praying. The entire intervention program consisted of ten sessions: one session for the introduction of the program and nine sessions of DBT skills training. All nine sessions were given in 3 weeks with three sessions each week.

The intervention program consisted of two types of training: skills training and skills reinforcement training. The duration of each session was between 45 and $60 \mathrm{~min}$. Details of the program are found in Table 1 below.

Table 1

Details of Intervention Program

\begin{tabular}{cccc}
\hline Week & Day & Types of Training & Module \\
\hline Preparation & Friday & Introduction & Introduction \& Learning Contract \\
& Monday & Skills Training & Mindfulness: State of Mind and Breathing Exercise \\
1 & Wednesday & Skills Training & Mindfulness: Chain Behavior \\
& Friday & Skills Reinforcement Training & Mindfulness: exercise about prior material \\
& Monday & Skills Training & Mindfulness: 'Check-Your-Tummy' Exercise \\
2 & Wednesday & Skills Training & Mindfulness: 'One Thing at One Time' \\
& Friday & Skills Reinforcement Training & Mindfulness: exercise about prior material \\
& Monday & Skills Training & Distress Tolerance: STOP \\
3 & Wednesday & Skills Training & Distress Tolerance: Exercise on The Spot and Praying \\
& Friday & Skills Reinforcement Training & Distress Tolerance: exercise about prior material \\
\hline
\end{tabular}

Activities in each session were based on the standard DBT skills training structure but with two added activities. The additional activities were a review about prior material before giving the new material and an evaluation of the new material at the end of the session. Therefore, the modified structure that was used in the program consisted of (1) mindfulness practice (as a warm up to begin the session), (2) homework review, (3) prior material review, (4) presentation of the new material, (5) "wind-down" (describing feelings), and (6) evaluation of the material and homework. In the skills reinforcement training, the participant was asked to do a worksheet, which allowed him to apply these skills in real-life situations. Besides making some changes in the training structure, some modifications were also made in the delivery method, including simplifying the language and using the following tools: videos and props as presentation materials, diary cards with pictures, emotion regulation skills cards, agenda book, and stickers as rewards.

Parents and teachers were included in this study to support the progress of the participant's emotion regulation skills. This supporting intervention consisted of two formal sessions, namely, one session with two teachers and the other with participant's mother. Teachers and the mother were taught about the skills to be given to the participant. The session was held before the skills training began. Informal meetings with teachers and the mother were also held during the intervention phase to provide information about things related to the development of the participant's emotion regulation skills. The fidelity of the intervention was accomplished by using a skills training module that was qualified by a panel of experts. The skills training module describes the steps and details involved in the intervention program. No standardized methods were used in this study.

\section{Analysis}

Observational data were analyzed through visual and statistical analyses. Visual analysis was conducted by comparing the percentages of emotion dysregulation response among the baseline, 
intervention, and evaluation phases. Statistical analysis was used to determine the significance of the intervention by comparing the percentage of emotion dysregulation response between the baseline and evaluation phases. Wilcoxon signed rank test was used because this technique is adequate to determine an intervention's impact with a small sample size, such as a single-case study (Kazdin, 2011 as cited in Tate et al., 2016). The study also used Wilcoxon signed rank test to evaluate the significance of the intervention as measured by ERC. The participant's progress during the intervention was also considered an evaluation of the study outcome. Qualitative data such as participant's knowledge and ability about emotion regulation skills, attention, cooperativeness, and emotions during the sessions, and other information related to the intervention were added to enrich the analysis.

\section{Results}

The interventions consisted of an intervention program to the participant (student with BIF), and they were supported with sessions with the parents and teachers. The program was conducted as planned in the procedure: one introduction session and nine intervention sessions. Each session was held after school hours and lasted for 40-60 min. No changes were made in the content of the materials, but there were some modifications in the methods used to deliver the materials, such as delivering materials through illustrative video. These changes were made to maintain the participant's motivation.

Sessions with the parents and teachers were also completed as planned. From teachers' reports, they found an effective way to react when the participant showed the challenging behavior (e.g., remind him to use the skills by showing the skills card and direct him with a calm voice and minimal verbal instructions). However, they often resorted to the "old" ways, which worsened the participant's emotion dysregulation, such as threatening him by filming his misbehavior or scolding him with a high-pitched voice and fast-paced instructions. Meanwhile, the participant's mother was quite consistent in reminding the participant to practice the emotion regulation skills. She often told him to do the breathing exercise or "STOP" technique when he started to show the challenging behavior. Even so, parental support was also not as expected; during the intervention, the mother let the participant do his diary cards without her guidance. The absence of this guidance made the participant fill his diary cards carelessly, so the diary cards could not be used as a self-monitoring tool. The mother also used the "old" way to communicate with the participant (high-pitched voice, fast-paced instructions, verbal instructions only), even though she knew the more effective way to communicate with him.

In general, an increase in the participant's emotion regulation skills was observed by the decreasing emotion dysregulation response during the intervention and evaluation phases upon comparing with the baseline (see Figure 1). As observed during the intervention, the participant gained knowledge and skills about emotion regulation concepts being taught. During the sessions, the participant was able to use breathing exercise when he experienced stressful conditions, although he still needed direct instructions to do so. 


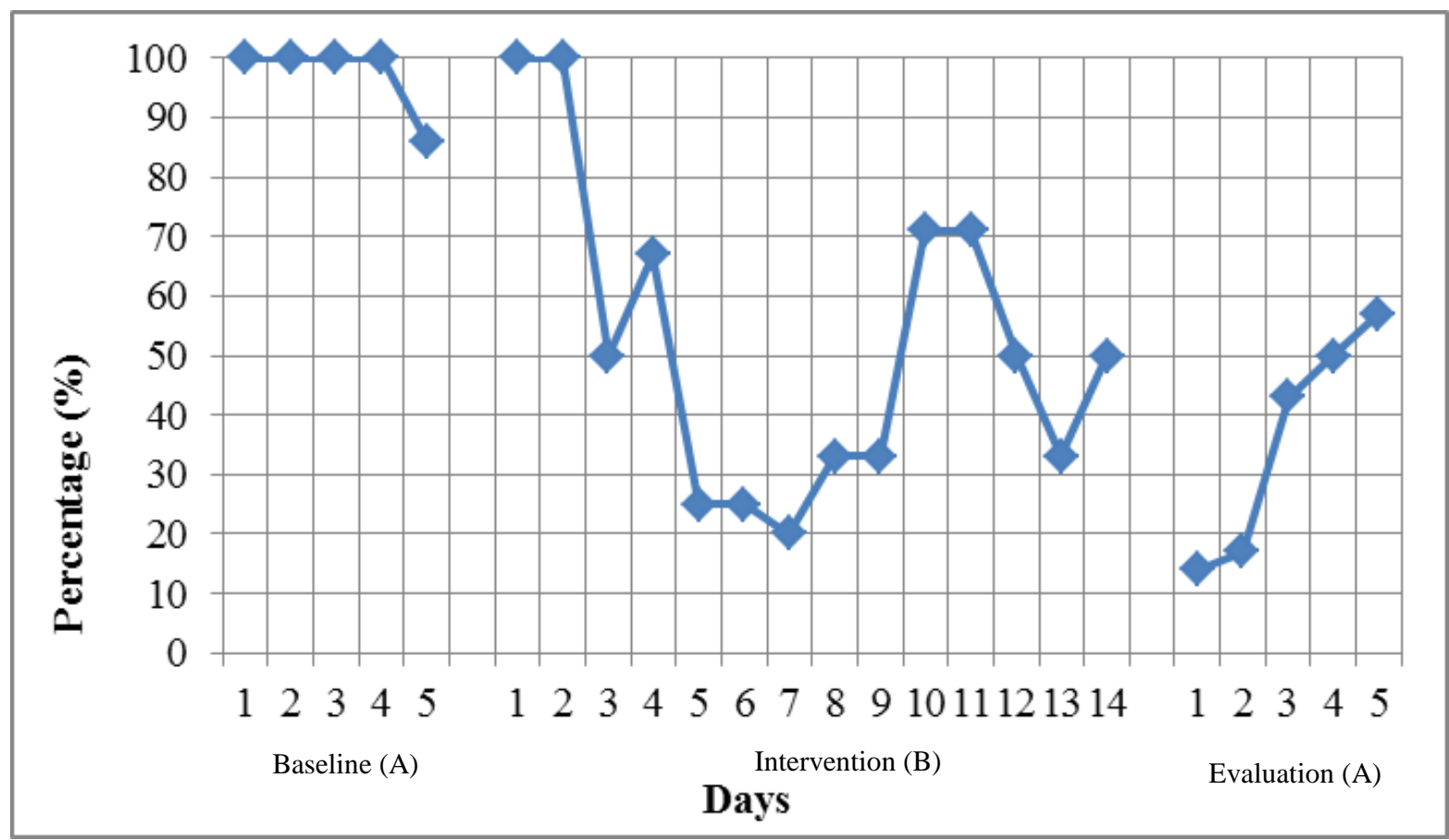

Figure 1. Percentage of emotion dysregulation response

In the graph above, days $1-5$ in the intervention phase was the first week, days 6-9 was the second week, and days 10-14 was the third week. Figure 1 shows that the emotion dysregulation response in the intervention and evaluation phases was lower than that in the baseline phase. In one incident, the emotion dysregulation response can be verbal response only, physical response only, or both responses. The details of the participant's emotion dysregulation response are shown in the tables below.

Table 2

Percentage of Verbal Emotion Dysregulation Response

\begin{tabular}{|c|c|c|c|}
\hline \multirow{2}{*}{ Behavior } & \multicolumn{3}{|c|}{ Phase } \\
\hline & Baseline & Intervention & Evaluation \\
\hline Making disturbing voices & $47 \%$ & $0 \%$ & $15 \%$ \\
\hline Complaining with a loud voice & $65 \%$ & $31 \%$ & $18 \%$ \\
\hline Arguing with appropriate/neutral words & $74 \%$ & $7 \%$ & $3 \%$ \\
\hline Arguing with inappropriate/aggressive words & $29 \%$ & $2 \%$ & $0 \%$ \\
\hline Screaming & $24 \%$ & $11 \%$ & $15 \%$ \\
\hline
\end{tabular}

Table 3

Percentage of Physical Emotion Dysregulation Response

\begin{tabular}{lccc}
\hline \multicolumn{1}{c}{ Behavior } & \multicolumn{2}{c}{ Phase } \\
\cline { 2 - 4 } & Baseline & Intervention & Evaluation \\
\hline Hitting the table (with hands or other objects) & $9 \%$ & $5 \%$ & $12 \%$ \\
Kicking the table/floor & $13 \%$ & $0 \%$ & $6 \%$ \\
Beating his own head (with hands or other objects) & $6 \%$ & $0 \%$ & $0 \%$ \\
Gripping objects but not throwing it or using it to attack people & $6 \%$ & $2 \%$ & $0 \%$ \\
Showing attacking gesture (no touching) & $22 \%$ & $7 \%$ & $6 \%$ \\
Throwing objects & $6 \%$ & $0 \%$ & $3 \%$ \\
Attacking people & $22 \%$ & $7 \%$ & $3 \%$ \\
\hline
\end{tabular}


Tables 2 and 3 show that the participant's emotion dysregulation was more verbal than physical. Table 2 reveals a substantial decrease both in the frequency of the dysregulation and the variation in the dysregulation after the intervention began. When experiencing stressful situations, the participant still complained with a loud voice $(31 \%)$ but rarely showed other types of verbal dysregulation. These changes were also evident in the occurrence rate of his physical emotion dysregulation. Nevertheless, a slight increase was observed in "hitting the table" in the evaluation phase (see Table 3).

As shown in Figure 1, the emotion dysregulation response increased up to $71 \%$ in the third week (days 10-14). These changes could be influenced by the way a teacher responded to the participant's behavior. In that week, the participant was given/received many verbal criticisms, instructions with a high-pitched voice, and fast-paced talks. He was being threatened that his behavior would be reported to his mother, and his behavior was completely ignored in some incidents. The participant's emotion dysregulation response was shown more frequently than in the previous two weeks. Figure 1 also illustrates that the dysregulation response decreased (14\%) in the evaluation phase but increased again until the end of the phase. This finding was also shown by the increase in some verbal and nonverbal dysregulation response (see Tables 2 and 3 ). This increase was influenced by many incidents during the evaluation phase (the participant being grounded by parents, being promised that he would go to a book exhibition, studying outside of the class, and having a school holiday) and a teacher who responded by threatening the participant when he did not follow the teacher's instructions.

Aligned with the decreased percentage of emotion dysregulation response, the participant showed adaptive response when he experienced a stressful situation. He regulated his emotion by not giving any response (standing still), avoiding students who annoyed him, and cooperatively doing what he was told to do. Improvement of his emotion regulation skills was also demonstrated by the reduced intensity of his emotion dysregulation response. When he experienced stressful situations, the participant still complained and made noises but in a lowvolume voice or whisper.

The decreased emotion dysregulation response in Figure 1 was consistent with the results of the statistical analysis $(\mathrm{Z}=-2.023, p<0.05, \mathrm{r}=-0.64)$. The significant difference between the baseline and evaluation phases shows that DBT skills training is effective to increase emotion regulation skills in elementary school children with BIF. However, this finding is not aligned with the result of the ERC measurement, which showed no significant difference in emotion regulation skills between before and after the intervention $(Z=1.826, p>0.05, r=-0.65)$. The ERC result was influenced by incomplete instructions during the post-test, so this result was not used to determine the success of the study.

\section{Discussion}

The results of the study show that DBT skills training is effective to increase emotion regulation skills in elementary school students with BIF. The participant gained knowledge about the emotion regulation concepts and skills, which helped him cope with stressful situations. This result supports the findings of a previous study about DBT effectivity among individuals with intellectual disability. Furthermore, the results supported the original hypothesis that DBT is not 
only effective for adolescents and adults (Brown et al., 2013; Charlton \& Dykstra, 2011; Florez $\&$ Bethay, 2017) but also for school-aged children with intellectual disability. These outcomes can be achieved by adapting the module, as suggested by McNair et al. (2016) that DBT skills training requires some basic modifications to meet the needs of a client with intellectual disability. These modifications help them apply a dialectical perspective without the struggle of understanding the abstract concept.

The materials in this study were delivered in visual, auditory, and kinesthetic modalities, such as using videos, yoga exercises, and breathing exercises. This method of delivery was consistent with the findings of Lew et al. (2006), who reported that materials should be presented in various modalities so the client can understand the information being taught. Information should be delivered in a language the participant can understand (Charlton \& Tallant, 2003). Therefore, in this study, abstract concepts were simplified into words that were familiar to the participant. For example, the term "emotion regulation" was changed to "self-control." The stories used in the video were also incidents that had happened to the participant.

In vivo practice helps the client generalize the skills being taught (Florez \& Bethay, 2017). One of the ways that can bring up an in vivo condition is by stimulating negative emotions through a chain behavior practice (Koerner, 2013). In this study, in vivo conditions were brought up through discussions about the participant's behavior in the classroom, confrontations when he lied, and refusals when he asked to watch a video or get a sticker. When he started to show emotion dysregulation, he was directed to do the breathing exercise until he did the exercise and calmed down. This kind of direction was also mentioned by Koerner (2013), who discussed micro-orienting; specific instructions about how to do a particular treatment task help participants work with their emotion dysregulation and information processing problems during a session (p. 573). This ability is necessary when dealing with clients that are prone to show emotion dysregulation, such as clients with intellectual disability. A client's successes in practicing mindfulness in DBT sessions cannot be separated from the therapist's mindfulness practice in his or her own life (Kabat-Zinn as cited in Hooker \& Fodor, 2008). This concept was demonstrated in the study from the ways the participant responded to the instructions. Instructions that were given mindfully helped the participant focus his attention. Otherwise, he would not behave cooperatively when the therapist was judgmental and talked emotionally. This finding shows that practicing mindfulness is necessary for therapists who want to teach mindfulness to their clients.

In dealing with clients with intellectual disability, Baillie and Slater (2014) stated that clients with intellectual disability often do things only because their caregiver told them to do it; therefore, they may have difficulties in developing a sense of commitment. In the study, the participant underwent difficulty in sticking to the learning contract that he agreed on at the first session (e.g., starting each session on time and being cooperative). In spite of this problem, he went through all the sessions because he knew that his parents had an agreement with the therapist about his participation in the program. One alternative that can be applied when working with clients in this population is to give them trial sessions before the long-term program begins (Baillie \& Slater, 2014). Thus, they have a chance to experience DBT and make a more informed decision about whether or not to commit to the DBT program. 
Environmental support in helping clients practice their emotion regulation skills in everyday life is the main factor that leads to the success of implementing DBT among clients with intellectual disability (Baillie \& Slater, 2014). Therefore, the way different environments support the client should be consistent from one environment to the other. By contrast, there were inconsistencies in how the therapist, teachers, and parents communicated with the participant. One of the findings in this study is that the participant behaved cooperatively when he received a slowpaced instruction delivered with a low-pitched, low-volume voice and calm intonation, and with both verbal and gestural forms, or gestural form only. However, teachers and the participant's mother communicated in the opposite ways. Moreover, teachers did not encourage the participant to practice the skills, and they responded to the participant's dysregulation with threats or verbal instructions. Aligned with the teachers' reactions, Green and Baker (2011) stated that clients with intellectual disability learn negative emotions more easily than positive ones. Negative responses from the teachers, combined with a lack of appreciation, reinforced the participant's dysregulated behaviors and his emotion dysregulation intensified.

The importance of a synthesis between acceptance and change in DBT means that a client must accept his or her emotions first before changing it to a more adaptive response (Linehan, 2015). Moreover, in dialectical perspectives, one's emotion dysregulation is influenced by the invalidating environment. In this study, the role of the invalidating environment was shown by the increases in dysregulation responses during the third week of intervention and evaluation phases. Threats and punishments given by teachers and the mother limited the participant's acceptance process and made it difficult for the participant to change his behavior.

Given the importance of validation from the environment, the client's environments have to show validation toward the client that he or she is doing the best he or she can do (Linehan, 2015). Therefore, the supporting intervention to the environment should emphasize the importance of environmental validation in developing the client's emotion regulation skills (Lew et al., 2006). In the study, the intervention for the teachers and parents only consisted of an explanation and practice about the emotion regulation skills. For future research, more structured and formal intervention for the client's environment with emphasis on the importance of validation should be considered when using DBT to treat clients with intellectual disability. When the environment becomes sufficiently aware to validate a client's condition, he or she will give positive reactions and help the client to regulate his or her emotion, instead of showing negative reactions as the teachers and mother did in this study.

Another limitation is influenced by the methodologies used in this study. The A-B-A design made it difficult for us to determine the effectivity of the intervention. Therefore, future research should consider using the A-B-A-B design for optimal outcomes. The implementation of DBT can be optimized by using the complete four modules of DBT skills training with a long duration. The findings in this study led to the need for further studies with more systematic and strict methodologies. However, the study extended previous findings by showing that DBT skills training is a promising therapy to increase emotion regulation skills in clients with intellectual disability, including school-aged children with BIF. 


\section{References}

Baglio, G., Blasi, V., Intra, F.S., Castell, I., Massaro, D., Baglio, F., ... Marchetti, A. (2016). Social competence in children with Borderline Intellectual Functioning: Delayed development of theory of mind across all complexity levels. Frontiers in Psychology, 7, 1-10, doi: 10.3389/fpsyg.2016.01604

Baillie, A. Slater, S. (2014). Community dialectical behavior therapy for emotionally dysregulated adults with intellectual disabilities Advances in Mental-Health and Intellectual Disabilities, 8(3), 166-173, doi: 10.1108/AMHID-05-20130033

Boulanger, M. (2015). Emotion regulation in children with Borderline Intellectual Functioning: Exploring the self-regulatory profiles of an understudied population (Master's thesis, McGill University, Montreal, Quebec Canada). Retrieved from http://digitool.library.mcgill.ca/webclient/StreamGate?folder_id=0\&dvs=1507731872009 468

Brown, J.F., Brown, M.Z., \& Dibiasio, P. (2013). Treating individuals with intellectual disabilities and challenging behaviors with adapted dialectical behavior therapy. Journal of Mental Health Research, 6, 280-303. doi: 10.1080/19315864.2012.700684

Charlton, M., Dykstra, E.J. (2011). Dialectical behavior therapy for special populations: Treatment with adolescents and their caregiver. Advances in Mental Health and Intellectual Disabilities, 5(5), 6-14. doi:10.1108/20441281111180619

Charlton, M., Tallant, B., (2003). Trauma treatment with clients who have dual diagnoses: Developmental disabilities and mental illness. Presented in the National Child Traumatic Stress Network, Colorado, USA. Retrieved from http://citeseerx.ist.psu.edu/viewdoc/download?doi=10.1.1.495.7112\&rep=rep1\&type=pdf

Davis, E.L., Levine, L.J. (2013). Emotion regulation strategies that promote learning: Reappraisal enhances children's memory for educational information. Child Development, 84(1), 361-374. doi:10.1111/j.1467-8624.2012.01836x

Florez, I.A., Bethay, J.S. (2017). Using adapted dialectical behavioral therapy to treat challenging behaviors, emotional dysregulation, and generalized anxiety disorder in an individual with mild intellectual disability. Clinical Case Studies, 1-16. doi:0.1177/1534650116687073

Graziano, P.A., Reavis, R.D., Keane, S.P., \& Calkins, S.D. (2007). The role of emotion regulation and children's early academic success. Journal of School Psychology, 45(1), 3-19. doi:10.1016/j.jsp.2006.09.002

Green, S., Baker, B. (2011). Parents' emotion expression as a predictor of child's social competence: Children with or without intellectual disability. Journal of Intellectual Disability, 55(3), 324-338. doi:10.1111/j.1365-2788.2010.01363.x

Hooker, K.E., Fodor, I.E. (2008). Teaching mindfulness to children. Gestalt Review, 12(1), 75-92. Retrieved from http://ww.gisc.org/gestaltreview/documents/TeachingMindfulnesstoChildren.pdf.

Jankowska, A. (2016). Towards a framework for psychological resilience in children and adolescents with Borderline Intellectual Functioning. Polish Psychological Bulletin, 47(3), 289-299. doi:10.1515/ppb-2016-0035

Karande, S., Kanchan, S., \& Kulkarni, M. (2008). Clinical and psychoeducational profile of children with Borderline Intellectual Functioning. Indian Journal of Pediatrics, 75, 795-800. doi: 10.1007/s12098-008-0101-y

Koerner, K. (2013). What must you know and do to get good outcomes with DBT?. Behavior Therapy, 44, 586-579. doi: 10.1016/j.beth.2013.03.005

Lew, M., Matta, C., Tripp-Tebo, C., \& Watts, D. (2006). Dialectical behavior therapy (DBT) for individuals with intellectual disabilities: A program description. Mental Health Aspects of Developmental Disabilities, 9(1), 1-13. Retrieved from http://gra.midco.net/pdkolstoe/MHS/DownloadResources/MHADD06-DBT-IDDPgmDesc.pdf

Linehan, M.M. (2015). DBT skills training manual $2^{\text {nd }} E d$. New York, NY: Guildford Publications.

McClure, K.S., Halpern, J., Wolper, P.A., \& Donahue, J.J. (2009). Emotion regulation and intellectual disability, Department of Psychology, LaSalle University, $\quad$ Philadelphia. $\quad$ Retrieved from https://www.researchgate.net/publication/263763300_Emotion_Regulation_and_Intellectual_Disability

McMain S., Korman, L.M., \& Dimeff, L. (2001). Dialectical behavior therapy and the treatment of emotion dysregulation. Psychotherapy in Practice, 57(2), 183-196. doi: 10.1002/1097-4679(200102)57:2<183::AID-JCLP5>3.0.CO;2-Y

McNair, L., Woodrow, C., \& Hare, D. (2016). Dialectical behavior therapy (DBT) with people with intellectual disabilities: A systematic review and narrative analysis, Journal of Applied Research in Intellectual Disabilities 2016, 3-18. doi:10.1111/jar.12277

McWilliams, J.L. (2014). The evaluation of the transformers programme: An emotion regulation programme for people who have an intellectual disability. (Doctoral Dissertation. Massey University, Wellington, New Zealand). Retrieved from https://mro.massey.ac.nz/bitstream/handle/10179/6537/02_whole.pdf?sequence=2\&isAllowed=y

Nickelson, C.A. (2013). Key elements of dialectical behavior therapy. Master of Social Work Clinical Research Papers. Retrieved from http://sophia.stkate.edu/msw_papers/241

Shaw, S.R. (2010). Recuing student from the slow learner trap. Principal Leadership, 10(6), 12-16. Retrieved from https://www.nasponline.org/Documents/.../Slow_Learners_Feb10_NASSP.pdf

Shields, A., Cicchetti, D. (1997). Emotion regulation among school-age children: The development and validation of a new criterion Q-sort scale. Developmental Psychology, 33(6), 906-916. doi: 10.1037//0012-1649.33.6.906

Sturmey, P. (2004). Cognitive therapy with people with intellectual disabilities: A selective review and critique. Clinical Psychology and Psychotherapy, 11, 222-232. doi: 10.1002/cpp.409

Van Nieuwenhuijzen, M., Vriens, A. (2012). (Social) cognitive skills and social information processing in children with mild to Borderline Intellectual Disabilities. Research in Developmental Disabilities, 33, 426-434. doi: 10.1016/j.ridd.2011.09.025 
Whitaker, S. (2001). Anger control for people with learning disabilities: A critical review, Behavioral and Cognitive Psychotherapy, 29, 277-293. doi: 10.1017/S1352465801003022 